\title{
Clima organizacional y valores: indicadores de necesidades y limitaciones para el cambio Caso: Colegio de Posgraduados.
}

\section{Organizational climate and values: needs indicators and change limitations: Postgraduate College Case}

\author{
Concepción Sánchez Quintanar ${ }^{1}$ \\ Profesora Investigadora \\ Colegio de Posgraduados \\ Montecillo, Texcoco Edo. de México \\ 2con.sanchez@gmail.com \\ José Luis García Cue ${ }^{2}$ \\ Profesor Investigador Asociado \\ Colegio de Posgraduados \\ Montecillo, Texcoco Edo. de México \\ jlgcue@colpos.mx
}

Recibido: 10 agosto 2012 Aceptado: 15 de julio Corregido: 29 de noviembre

Resumen $^{3}$ : En el contexto de los cambios que modernizan a las universidades y centros de educación superior en América Latina, este artículo presenta la problemática del caso del Colegio de Posgraduados (CP). Con el fin de entender sus sistemas de organización y comportamiento, se trabaja en el análisis del Clima Organizacional (CO) y las características de los académicos (valores, actitudes sociales y liderazgo). Este trabajo es la primera etapa, en relación con el análisis de las relaciones del CO y los valores. Los objetivos fueron: Comprender la dinámica entre las dos variables; identificar limitaciones de desarrollo y sugerir procedimientos de apoyo. Se trabajó con una muestra de 90 académicos, de todos los niveles. Se aplicaron dos escalas, una de CO y otra de Valores, los perfiles obtenidos fueron de

1 Licda. en Psicología Universidad Autónoma de México, 1971. MC. Divulgación Agrícola, Colegio de Posgraduados (CP),1973. Mtra y Dra. en Orientación y Desarrollo Humano, Universidad Iberoamericana, 1982, 1984. Profesora Asociada, Universidad Autónoma Metropolitana 1981, 1984. Directora Fundadora de la Escuela de Psicología Universidad Pedro de Gante 1989 - $1991 . \quad$ Profesora Investigadora Titular, en la Especialidad Desarrollo Rural CP 1999 a la fecha. Miembro del Sistema Nacional de Investigadores Nivel I desde 1992. Vice Presidenta -México de la Asociación Latinoamericana de Sociología Rural 1994 -1998. Libros Publicados: Método de Freire en Programas de Desarrollo Rural 1990, Desarrollo de Grupos e Integración personal. Editorial Contraste 1986. Psicología en Ambiente Rural, Plaza y Valdés 2010. Coordinadora de libros: Psicología Alternativa en América Latina, AMAPSI 2006. Estado, Política y Institucional en el Sector Rural de América Latina 2000. Líneas de investigación: Análisis Organizacional en el Sector Rural. Psicología en Ambiente Rural. Publicación de artículos derivados de las Líneas de investigación y docencia en maestría y doctorado.

2 MC. Estadística y Cálculo por el Colegio de Posgraduados. Dr en Educación Universidad Nacional de Educación a Distancia, UNED, España AÑO. Tesis: Los Estilos de Aprendizaje y las Tecnologías de la Información y la Comunicación en la Formación del Profesorado. Profesor Investigador Asociado del Colegio de Postgraduados (CP) en de Estadística en el Campus Montecillo. Coordinador del Proyecto Internacional de Investigación en Educación de Gestión del Conocimiento en México. Su Línea de Investigación Estilos de aprendizaje y diseño de Educación en Línea, para cursos de Estadística y Probabilidad lo desarrolla en la FES - Universidad Nacional Autónoma de México. En el Colegio de Posgraduados el Proyecto de Transferencia de Tecnología vía internet para los cursos Estadística, Probabilidad, Diseños Experimentales y Paquetes Estadísticos. Miembro de los Comités Editoriales y de Redacción de la Revista de Estilos de Aprendizaje-Learning Styles Review (www.learningstylesreview.com) y Comunicaciones del Instituto de Socioeconomía Estadística e Informática. Ha participado en la Publicación de 4 libros sobre Informática y Lenguajes de Programación. Publicación de diferentes artículos sobre Educación a Distancia, Integración de TIC en el Currículo de los Estudiantes. Correo electrónico: jlgcue@colpos.mx

3 Se reconoce y agradece al MC Raúl del Valle Paniagua Investigador Adjunto por la recolección y manejo de la información. 
nivel medio y medio-bajo respectivamente. La estadística Correlación de Pearson, identificó todas las relaciones posibles entre los elementos de las dos escalas. Los resultados más importantes fueron: Los perfiles de las escalas revelaron dificultades en los académicos para percibir la situación organizacional, y formas débiles para manifestar sus valores. Las principales limitaciones de la institución se determinan en las dimensiones Estructural, Estándares y Responsabilidad. Las limitaciones del personal académico son el deterioro en sus capacidades volitivas, comportamientos evasivos de responsabilidades y agresión generalizada. Se ofrecen sugerencias para superar las limitaciones del CO y desarrollo del personal académico.

Palabras claves: Modernidad, educación superior, cambios, limitantes.

Abstract: Within the context of modernizing changes for universities and higher education institutions in Latin America, this paper presents the problem of specific Postgraduates Colleges. In order to understand their organization and behavior problems, researchers are working on the analysis of Organizational Climate (CO) and the characteristics of academic staff (values, social attitudes and leadership). This work is the first step, analysis of the relations of $\mathrm{CO}$ and on the Values. The objectives were: To understand the dynamics between the two variables; to identify development limitations; and to suggest procedures for support. The sample consisted of 90 academics at all levels. Two scales were applied, one for $\mathrm{CO}$, another one for values. The statistic Pearson correlation identified all possible relationships between the elements of the two scales. The most important results were: The profiles revealed difficulties in academics to perceive the organizational situation to express their values. The Structural, Standard and Liability dimensions determine the main limits of the institution. The limits of academic personnel are impairment in their volitional abilities; behaviors to avoid responsibilities and to diffuse aggression. Suggestions are offered to overcome the limitations for CO and to improve academic personnel.

Keywords: Modernity, higher education, changes, limiting.

\section{Introducción}

Este trabajo se realizó en el Colegio de Posgraduados (CP), corresponde a una investigación mayor cuya meta es conocer el potencial de cambio que tiene la institución, tanto en el nivel organizacional como en el humano. El contenido de este artículo corresponde a la primera fase y tiene los siguientes objetivos:

a. Comprender la dinámica de las relaciones entre el Clima Organizacional de la institución y los Valores del personal académico.

b. Identificar los límites de desarrollo que se derivan de la dinámica entre el Clima Organizacional de la institución y los Valores del personal académico

c. Sugerir procedimientos de apoyo al potencial humano del cuerpo académico y su organización.

Los procesos de cambios estructurales y económicos de origen neoliberal en los países más desarrollados, han inducido transformaciones en todo el orbe social, en especial en los países de menos desarrollo. Los que aquí nos interesan son los producidos en América Latina en especial en universidades e instituciones de enseñanza superior, quienes enfrentan la necesidad de modernizarse y responder a las demandas laborales de personal calificado 
y profesionales especializados, que se entiende atenderán las demandas económicas y productivas de los cambios.

En la zona latinoamérica del continente los sistemas de educación superior pasaron por una etapa de resistencia al cambio, hasta identificar las transformaciones requeridas, para no quedar a la saga del conocimiento que ahora se produce con gran rapidez y cantidad. "En la educación de posgrado, hay más dificultades para el cambio por que tienen mayor tiempo empleando otros modelos de enseñanza" (Izarra y Escobar, 2007, p.172); sin embargo los autores reconocen las ventajas de procedimientos didácticos modernos.

Algunas universidades han atendido más al cambio estructural, influidas por los modelos de satisfacción al cliente, pensando que el cambio en el aprendizaje será consecuencia del organizacional. Esta perspectiva incluye más las vertientes sociológica, administrativa y relaciones humanas que la orientación pedagógica (Quintero et al., 2010). En los procesos de modernización educativa de las universidades de América Latina se pueden distinguir dos etapas:

\section{$1^{\text {a }}$. Etapa. Reacciones frente a la modernización.}

Al inicio del siglo XXI la atención de las universidades se centraba en la disyuntiva entre preservar y acrecentar la cultura, el desarrollo integral del ser humano, la solución de problemas del conocimiento dadas las condiciones de deterioro ambiental y social; o convertirse en vendedoras de conocimientos y formadoras con eficiencia de recursos humanos para los grandes capitales (Mollis, 2002). El Estado ha querido influir en las universidades "en forma vertical y autoritaria sobre las bondades de las fuerzas naturales del mercado" (Porter, 2004, p. 588). Otras estructuras universitarias se han hecho verticales y burocráticas, anulando la individualidad para la creatividad y transformación (Acosta, 2005). Se deja de valorar la autonomía como facilitadora del pensamiento creativo y sensible a los problemas sociales. Las demandas del mercado ocupacional piden racionalidad instrumental y eficiente para consorcios privados (Lopera, 2004) "Acrecentándose la dependencia del exterior, por el escaso desarrollo científico y tecnológico." (Salas, 2007, p. 29).

\section{$2^{\mathrm{a}}$. Etapa. Estudios de casos}

Entre los años del 2000 al 2010 proliferaron diferentes estudios de caso, descriptivos, relacionales de variables o globales e inclusivos de factores externos que inciden en los procesos de cambio, solo se citan algunos de los más representativos:

Limitaciones de presupuesto no previstas provocan problemas de insolvencia para renovar al profesorado y retiros decorosos, derivándose otras necesidades urgentes, entre ellas: formación e incorporación de profesionales jóvenes a la docencia e investigación, y exceso sobrecarga de trabajo en el personal contratado (Rodríguez, Urquidi y Mendoza, 2009; Hirsh, 2008; Pedró, 2006).

El salario, estabilidad laboral, vocación académica e interés en la formación de nuevas 
generaciones, proporcionan satisfacción laboral al personal académico. Causan renuncia en el 50\%, limitación en decisiones que afectan su trabajo, falta de tiempo para actualizarse, la calidad de los estudiantes, falta de apoyo administrativo y sobrecarga de trabajo. El personal docente más joven es el menos satisfecho, ingresaron sin plaza, por renovación de contrato y sin apoyo administrativo (Padilla, Jiménez y Ramírez, 2008).

El personal docente tiene baja percepción de los cambios, los que ve son administrativos y de gestión; lo que menos percibe son los del modelo pedagógico y el nuevo marco normativo (Quintero et al. 2010). La explicación que el autor da a este hecho es que los procesos de cambio "llegaron del exterior, sin plan estratégico o modelo de gestión efectivo; por ello, los efectos y la percepción que los docentes tienen de ellos son limitados; sin embargo, han generado transformaciones y focos de interacción entre maestros" (p. 37).

Las universidades tienen contradicciones y duplicidad de acciones, entre "los posicionamientos teóricos o documentos básicos, y la práctica, lo que requiere de un nexo entre formación y el análisis de la realidad" (Esquivel, 2008, p. 13). Es común en la doble evaluación docente, la realizada por estudiantes y la académica del Plan de Calidad de las Universidades, que no coincidan los indicadores (Muñoz, Ríos y Abald, 2002). La estrategia exitosa que reporta para producir el "cambio del paradigma centrado en la enseñanza, hacia el aprendizaje autónomo de los alumnos, fue proporcionarle a todos los docentes capacitación constante durante el proceso" (De Miguel, 2006, p. 5) .

\section{Antecedentes del caso Colegio de Posgraduados}

El Colegio de Posgraduados (CP) se fundó en 1959, único posgrado en Ciencias Agrícolas que tiene México, integra docencia, investigación y vinculación con muy diferentes tipos de organismos sociales. Su ubicación en la estructura nacional es compleja, por que depende de la Secretaria de Agricultura, Ganadería, Desarrollo Rural, Pesca y Alimentación (SAGARPA); de su misión y visión derivan las actividades sustantivas antes mencionadas. Los objetivos básicos del CP son la generación de conocimiento científico a nivel básico, aplicado y tecnológico, enfocado a resolver los problemas de producción y ambientales de la población rural; y formar profesionales de alto nivel académico para este sector. Debe combinar la docencia e investigación con la vinculación, demanda que conduce a los docentes a relacionarse con organismos académicos, productivos, de productores, instituciones del gobierno a diferentes niveles e incluso del sector privado.

El CP es un sistema de 8 campus distribuidos en diferentes regiones atendiendo las necesidades climáticas predominantes del país. Desde su establecimiento ha sido sensible a los cambios económicos y del desarrollo, por ello periódicamente revisa la calidad de sus resultados. Solo se mencionarán los estudios relacionados con el factor humano: Seguimiento de egresados y necesidades de los organismos en donde trabajaban (González y Rendón, 1986 y 1999); causas de retraso en la graduación (CACM, 2005); sistemas de asistencia técnica (López, Villa y Badillo, 1983); recursos humanos de los Planes Regionales en etapas de modernización (Sánchez, 1984; Sánchez, Vázquez y Aguilar 1994); indicadores para evaluar 
actividades sustantivas (Polanco, 1992); análisis organizacional, (Starks, 2005) este trabajo tiene la limitante de que el director general escogió al personal académico de la muestra.

En el año 2003 el CP se convirtió en Centro Público de Investigación (CPI), y en el mismo año hubo una iniciativa de ley para desarticular varias instituciones, entre ellas a las de investigación y docencia del sector rural. Como medida para detener tal iniciativa se formó una (Comisión Mixta, 2004) cuyo objetivo fue evaluar la institución. La comisión estuvo formada por miembros de la Universidad de Wageningen, Holanda y por México integrantes del CP, el análisis abarcó las áreas administrativas y financieras, aportando sugerencias concretas para la modernización institucional.

No obstante el cambio del colegio a CPI, sigue dependiendo de la SAGARPA, sus directivos tienen posiciones de funcionarios públicos, dados esos roles sus decisiones afectan la vida académica, redujeron el personal de apoyo y secretarial teniendo los académicos que realizar algunas de sus labores, también los sustentos económicos para la investigación y docencia; eliminaron Cuerpos Colegiados reduciendo con ello la participaciones de los académicos en las decisiones, también detuvieron la homologación de su salario con sus pares de CPI de otras especialidades. Los académicos por su parte progresivamente asumieron algunas labores de apoyo o administrativas, lo que creó disgusto por el aumento de trabajo que los distrae de sus actividades fundamentales. Por estas causas se formó el Sindicato de Trabajadores Académicos.

Los cambios anteriores apoyados en nueva reglamentación que da preferencia a la estructura vertical, definieron el enfoque empresarial y la productividad privilegiada a la generación de recursos, principalmente económicos y el aumento en número de los "productos". Las innovaciones que los directivos establecen en enfoque y estrategias interfieren en la identidad académica y dificultad para tomar decisiones. En los primeros años de la reestructura, el CP calificaba en los parámetros de CPI, como "Excelente", paulatinamente ha bajado y llegado a ser "Buena".

\section{Problema de investigación}

La situación del CP descrita brevemente en el apartado anterior duró 13 años, período caracterizado por fuertes y sostenidas resistencias al cambio en una parte del personal académico; otra parte respondió al enfoque empresarial y diseño de agro negocios; y en mayor proporción a la defensa del salario e igualdad de apoyos para el desarrollo de las actividades sustantivas que tienen otros CPI. Otros aspectos muy importantes para la renovación del CP han sido el cese de funciones de los directivos y sus allegados por malos manejos y desvíos de presupuesto, y el logro de la homologación salarial. De tal suerte que la Comunidad Académica puede ahora abocarse a los cambios y actualizaciones que requiere, de donde se deriva el problema de conocer: ¿Cuáles son las capacidades y limitaciones organizacionales del CP y que relaciones tienen con los valores del personal académico? 


\section{Consideraciones Teóricas}

En el área multidisciplinaria del Desarrollo Organizacional (DO) se consideran como capacidades para el cambio la estructura organizacional y los procesos grupales, porque ambos inciden en la forma del comportamiento individual y grupal de los miembros de cualquier organismo social. Es necesario explicar que en México el radio de acción de los despachos o agencias de asesoría y conducción de DO, no abarcan al medio rural, en este se han adoptado algunas métodos y técnicas de forma aislada, no bajo diagnóstico ni como parte de un proceso nuevo de organización.

\subsection{Clima Organizacional}

Cuando se analiza el Clima Organizacional de alguna institución, hay conceptos que requieren de su justa apreciación como es el caso de la Cultura Organizacional, para cuya explicación se recurre a la semejanza y significado de cultura desde la perspectiva antropológica, refriéndose a todo lo desarrollado por el género humano; formas y reglas de vida, distribución de roles sociales, valores que rigen comportamientos, formas de transmitir y adaptar a nuevas generaciones a la sociedad constituida; tecnología, ciencia y legados históricos.

En la panorámica del DO, Cultura es un concepto más amplio que Clima, su diferenciación ha causado discusiones, hasta entenderse como complementarios. La Cultura Organizacional es el conjunto de valores, necesidades, expectativas, creencias, políticas y normas aceptadas y practicadas por las organizaciones. Desde las décadas de 1980 y 1990 se le considera con características tipificadoras del comportamiento del personal y la imagen del organismo social total (Quinn y Rohrbaugh, 1983; Schein, 1988; Pümpin y García, el 1993). Al presentarse como un concepto abstracto, la Cultura Organizacional ha dado como marco de comprensión, la relación entre ésta y el cambio, que puede ser un proceso natural o inducido, en donde intervienen los líderes (Schein, 2010). Estos personajes se identifican por el perfil individual, posición en la organización y relaciones con los miembros. Su ubicación organizacional se explica por personalizar las características de la cultura organizacional, ser reconocidas por sus seguidores al identificarlo como modelo de comportamiento aceptado por el organismo social.

Para diferenciar el Clima de la Cultura Organizacional, (Burton y Obel, 2004) enfatizaron que la Cultura es un patrón integrado de conocimiento humano, creencias y comportamiento que depende para manifestarse de las capacidades de aprendizaje. Las características del $\mathrm{CO}$, aun cuando revelen características de la Cultura Organizacional, se pueden describir y ser susceptibles de medición. La separación de los conceptos ha delimitado características y aportado claridad en cada uno promoviendo un sin número de investigaciones; las de Clima Organizacional forman dos corrientes:

- Las que valoran la percepción que los empleados tienen de los factores organizacionales internos y externos. Su importancia desde la psicología cognoscitiva es la percepción como la base del conocimiento y por ende del comportamiento. 
- Las que otorgan más peso a las interacciones del personal en las organizaciones, porque intervienen en la estructura y son un elemento ético, que de acuerdo con (Lemmergaad, 2003), se extiende a las dimensiones de valores y moralidad, relacionadas con la asimilación de valores y creencias de la cultura en donde se desenvuelve el organismo social.

La inclinación por el $\mathrm{CO}$ en los estudios contemporáneos es metodológica, porque se investigan características observables y medibles como variables - valores, autonomía individual, grado de imposición estructural, orientación al trabajo, consideración al trabajador, calidez y apoyo, entre otras (Burton y Obel, 2004).

Litwin y Stringer, 2003) dieron una perspectiva macro al análisis de la organización cuando definieron al CO como los atributos percibidos del organismo social y sus subsistemas o dimensiones, convirtiéndose en un reflejo de como el organismo se ocupa de sus miembros, grupos y problemas. El énfasis lo centran en los atributos percibidos del funcionamiento de los subsistemas (Gupta, 2008). Además, la escala que elaboraron Litwin y Stringer ha acumulado un soporte experimental por estudios de carácter diacrónico y sincrónico en muy diversas organizaciones y países (Koene, Vogelaar, y Soeters, 2002; Mano y Yiannis, 2006).

Para destacar la percepción de los integrantes de un organismo social, Litwin y Stringer subrayan la importancia de la subjetividad como centro de investigación en el CO, por la variabilidad que puede tener la percepción individual. En la actualidad la percepción subsiste como capacidad básica en el comportamiento organizacional, "las investigaciones realizadas sobre la percepción del CO, a nivel individual, y el CO mismo, están relacionadas con la satisfacción en el trabajo, aunque poco se ha investigado su importancia relativa" (Schulte, Ostroff y Kinicki, 2010. p. 646). La percepción también repercute en otro aspecto muy importante en las organizaciones, "entre el organismo social y cada miembro del personal y el contrato psicológico" (Epitropaki, 2012, p. 2).

\section{2. Cambios y valores}

Los cambios modernizadores, de origen externo que reciben los países de menos desarrollo, impactan su cultura al modificar en las sociedades paradigmas de comportamientos individuales y sociales, formados por las interacciones personales desde la niñez, lo que puede constituir la base de la resistencia al cambio. En el ámbito educativo los paradigmas son especialmente necesarios por que construyen valores, normas de comportamiento y orientación socializante de niños y jóvenes, capacitándolos para tomar decisiones culturalmente aceptadas. Desde esta perspectiva los valores son vitales en la esfera individual, familiar y organizacional.

Por lo anterior los valores se han estudiado a través de la historia desde los ángulos filosóficos, teológicos, psicológicos, teóricos y metodológicos, y en diferentes niveles de escolaridad. En la actualidad y en la educación superior se convierten en un tema inherente a todos los planteamientos de reforma y mejoramientos educativos, por que "la educación es por naturaleza, una cuestión de valores y un proceso de formación moral" (Barba, 2005: p. 9). 
En la actualidad las universidades han disminuido las discusiones relativas a la esencia del valor, intrínseco o adjudicado, o si se presenta en polaridades; en concordancia con la época, se quiere atender los reclamos sociales y proceder a la construcción de nuevos valores. En la actualidad podría constituir un valor el reconocimiento de las crisis sociales a partir de lo que se vive como lo hace Mercado (2004) "A partir que vemos en los medios de comunicación, de los diálogos que escuchamos en los diferentes lugares a donde asistimos, coloquialmente llegamos a sostener que la sociedad "se está pudriendo", "que se está desmoronando" "que está en crisis" "que ya no es como antes" "que se han perdido los valores." " (p. 136). Un reconocimiento de este calibre induce a enfrentar la realidad en la vivencia de los valores en la universidad y sus docentes, y encontrar uno de los problemas que sobre la ética profesional señala Pérez-Castro (2010) "El primero es que existe una confusión en los maestros entre ética filosófica, deontología y ética profesional, y la débil formación de los encargados de las asignaturas de formación ética" (p. 4).

Las decisiones diarias que toman los docentes en el contenido, metas y procesos pedagógicos, constantemente realizan el razonamiento y la jerarquización o priorización de las opciones susceptibles de elegir. Lo que constituye un apoyo suficiente para proponer “ como bases filosóficas de educación, procesos del desarrollo moral y psicológicos que aportan a la identificación de comportamientos precisos a los que se refieren los valores", (García y Pérez, 2005, p. 132).

Entre los problemas de los docentes para adoptar y provocar los valores contemporáneos requeridos, se identifican limitaciones de formación académica y personales, como su apego a lo conocido y lo seguro, y la falta de tiempo para actualizarse. La UNESCO citada por (Bárcenas, 2009), señala la conveniencia de que "la educación tenga las características de calidad para todos los niveles sociales, además de eficacia, eficiencia, relevancia, pertinencia y equidad" (p. 349). "La investigación educativa agrega a la pertinencia, normas y que los contenidos curriculares sean definidos por los sujetos de la educación en sus interacciones" (Bárcenas, 2009. p. 350).

La investigación educativa con aportaciones de la psicología ha identificado comportamientos convenientes para una mayor actividad entre los estudiantes, acuñando el concepto competencias, dada la asociación con producir, crear y organizar el medio en el que viven.

Por necesidades culturales se trabaja en la formulación de una ética profesional, generada por la experiencia de los profesores, que incluya temas y rasgos relevantes para formar su propio marco teórico (Hirsch, 2008 p. 2).

\section{Metodología}

Cuando se emplean escalas para apreciar comportamientos humanos, se obtiene la elección del entrevistado como una apreciación subjetiva, y otra objetiva cuando el mismo le da un peso numérico. Por ello se eligieron las escalas de CO de (Litwin y Stringer, 2003) y 
la escala de valores de (Allport, Vernon y Lindzey, 1970).

\subsection{Definiciones operativas.}

Clima Organizacional Es "el conjunto de propiedades del ambiente de trabajo, subjetivamente percibidas por los trabajadores el cual influencia su motivación y comportamiento" (Litwin y Stringer 2003: viii).

Valores "Los valores hacia el trabajo son las representaciones cognitivas de necesidades universales expresadas en metas transituacionales que se organizan en forma jerárquica y que se manifiestan en el entorno laboral" (Allport, Vernon y Lindzey, 1970, p. 1).

\subsection{Instrumentos}

La escala de CO está formada por seis Dimensiones de la vida de un Organismo Social: Estructura, Estándares, Responsabilidad, Reconocimiento Laboral y Apoyo. Su formato es tipo Likert de cuatro alternativas de respuestas: a) Definitivamente en desacuerdo; b) Inclinado al desacuerdo; c) Inclinado al acuerdo y d) Definitivamente de acuerdo. Los casos b) y c) se interpretan como tendencia a percibir o creer en la existencia de los comportamientos organizacionales que identifica la escala.

La escala de Valores mide seis tipos: Teórico, Económico, Estético, Social, Político, y Religioso. La persona que la responde, tiene que jerarquizar diferentes ítems de cada tipo de valor, las que constituyen opciones; así cada persona produce su propia escala. Esta escala se basa en la teoría de la personalidad de Allport, cuyos rasgos son las actitudes valorativas que distinguen al individuo como unidad y se aprecia como valor predominante el que se manifiesta de manera sobresaliente en el perfil de la escala, los demás contribuyen a describir a la personalidad de los entrevistados. Los trabajos de Allport continúan vigentes, (Arciniega y González, 2000) consideran “[...] la importancia actual de sus aportaciones empleándolos en sus trabajos, investigaciones y definición de valores" (p. 284). En otras investigaciones lo hacen, (Brewczynski y Douglas, 2006; Pierce et al., 2007; Jones, et. al. 2012).

\subsection{Población y Muestra}

La población fueron 522 académicos del CP de las distintas categorías académicas. La muestra se calculó de manera empírica apoyado en la fórmula de Santos et al. (2003:87-88) sobre muestreo aleatorio simple:

Donde

$$
n=\frac{Z_{1-\alpha / 2}^{2} N P Q}{\delta^{2}(N-1)+Z_{1-\alpha / 2}^{2} P Q}
$$

$$
\begin{aligned}
& \mathrm{N}=522 \text { profesores del } \mathrm{CP} \text { de distintas categorías académicas. } \\
& \delta \quad=0.10 \text { error máximo tolerado. } \\
& Z_{1-a / 2}=1.96 \text { para un valor del } 95 \% ; \quad \mathrm{p}=0.50 ; \mathrm{q}=1-0.50=0.50
\end{aligned}
$$


$\mathrm{Al}$ sustituir los datos en la fórmula se obtiene lo siguiente:

$$
n=\frac{(1.96)^{2}(522)(0.50)(0.20)}{(.10)^{2}(522-1)+(1.96)^{2}(0.50)(0.50)}=81.24
$$

Esto es, se necesitaron de al menos 82 personas académicas para una muestra representativa de la población de estudio, se trabajó con 91.

\subsection{Análisis Estadístico}

Los baremos internacionales de las escalas empleadas consideran tres niveles de respuestas: Calificaciones bajas entre 0-39, medias entre 40-69 y altas entre 70 - 100 . Para el análisis e interpretación la escala de $\mathrm{CO}$ el primer rango revela bajas capacidades de percepción de lo que se les interroga y por lo tanto de cambio; el nivel medio registra indecisiones, ambigüedades y divisiones en las percepciones, el último rango capacidades perceptuales altas y comprensión organizacional, que diagnostica participación positiva y clara en el cambio organizacional. La escala de Allport expresa tanto intensidad y claridad, así los rangos señalan medidas bajas, medias o altas en las dos cualidades.

También se emplearon estadísticos descriptivos, Análisis de la Varianza $(\alpha=0.05)$ con pruebas de comparación de medias de Tukey y Análisis de Correlación de Pearson

n ( $\alpha=0.05)$. Se usaron los programas SPSS V19.0 y SAS V9.3 para los cálculos.

\section{Resultados}

\subsection{Perfil de Clima Organizacional}

Las medias y desviaciones estándar de cada Dimensión Organizacional, se concentran en la Tabla 1; el Perfil de CO del Personal Académico es de nivel medio (Figura 1).

Tabla 1. Estadísticos descriptivos de Clima Organizacional

\begin{tabular}{llll}
\hline Dimensiones & N & Media & Des. Est. \\
\hline Estructura & 90 & 61.31 & 11.31 \\
Estándares & 90 & 61.18 & 13.78 \\
Responsabilidad & 90 & 60.68 & 14.03 \\
Reconocimiento & 90 & $\mathbf{6 4 . 8 1}$ & 14.28 \\
Apoyo & 90 & $\mathbf{6 6 . 8 1}$ & 10.57 \\
Compromiso & 90 & $\mathbf{6 6 . 5 0}$ & 7.74 \\
\hline
\end{tabular}




\section{Figura 1. Perfil del Clima Organizacional en el Personal Académico}

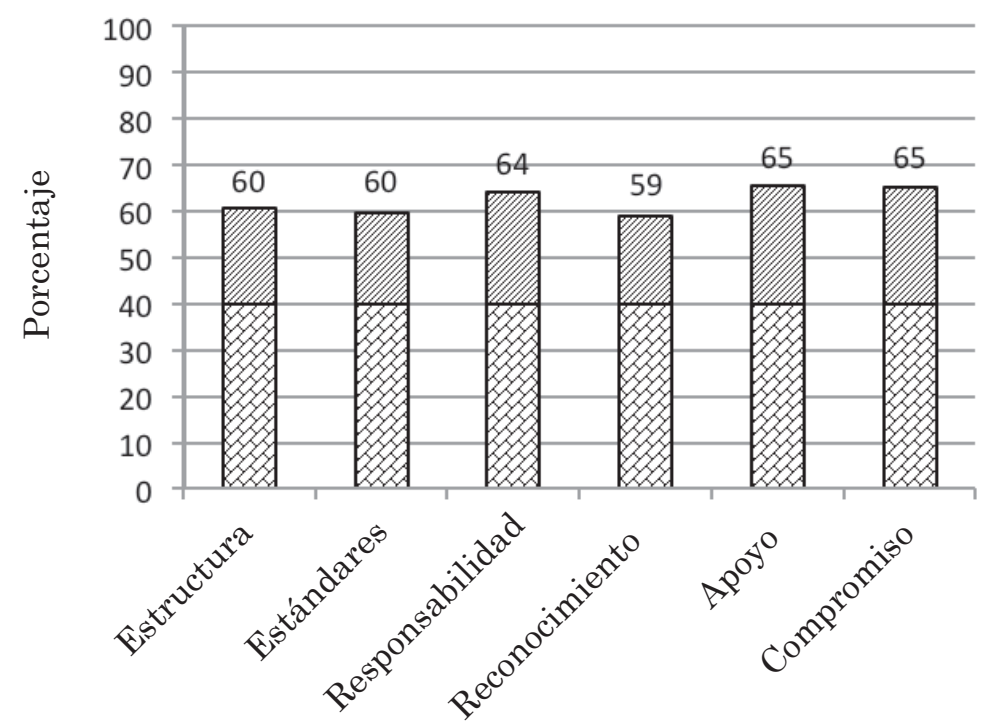

En las pruebas de comparación de medias de Tukey (Tabla 2) se distingue que las dimensiones Apoyo, Compromiso y Reconocimiento son estadísticamente similares, y de mayor puntuación que las Dimensiones Estructura, Estándares y Responsabilidad, también estadísticamente similares entre sí.

Tabla 2. Prueba de comparación de medias de Tukey.

\begin{tabular}{lll}
\hline $\begin{array}{c}\text { Dimensiones } \\
\text { Organizacionales }\end{array}$ & Media & Grupos \\
\hline$\underline{\text { Apoyo }}$ & 66.81 & $\mathrm{~A}$ \\
Compromiso & 66.50 & $\mathrm{~A} \mathrm{~B}$ \\
$\underline{\text { Reconocimiento }}$ & 64.81 & $\mathrm{~A} \mathrm{~B} \mathrm{C}$ \\
Estructura & 61.31 & $\mathrm{~B} \mathrm{C}$ \\
Estándares & 61.18 & $\mathrm{C}$ \\
Responsabilidad & 60.68 & $\mathrm{C}$ \\
\hline
\end{tabular}

Valor critico de Rango Estudentizado=4.04.

Diferencia Minima Significativa $5.18 \%$

\subsection{Perfil de Valores}

La Tabla 3 contiene las medias y desviaciones estándar de los Valores estudiados; el Perfil del Personal Académico (Figura 2), corresponde a un nivel medio - bajo. 
Tabla 3. Estadísticos descriptivos de Valores

\begin{tabular}{llll}
\hline Valores & $\mathrm{N}$ & Media & Desv. típ. \\
\hline Teórico & 90 & 51,883 & 6,6961 \\
Económico & 90 & 41,428 & 7,0386 \\
Estético & 90 & 39,350 & 7,2144 \\
Social & 90 & 38,561 & 7,0176 \\
Político & 90 & 38,811 & 5,4027 \\
Religioso & 90 & 29,967 & 7,9654 \\
\hline
\end{tabular}

Figura 2. Perfil de Valores Personal Académico

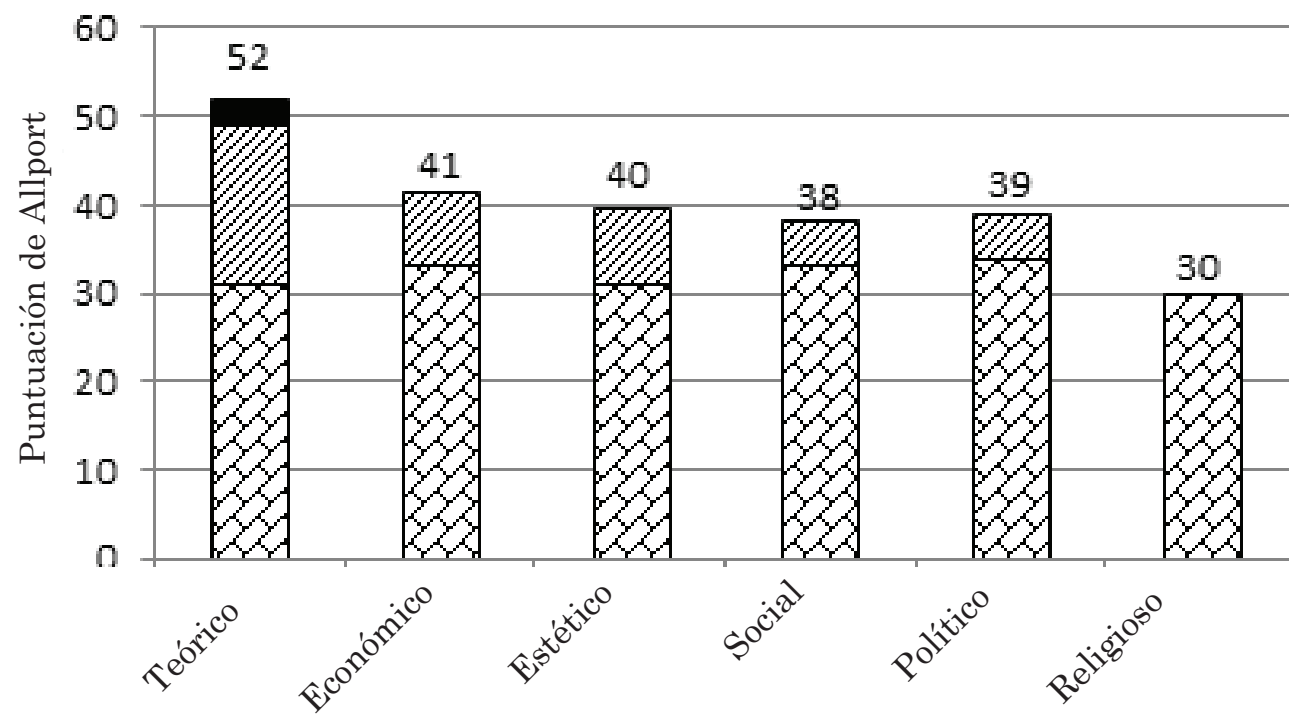

En las pruebas de comparación de medias de Tukey (Tabla 4) se distinguen tres diferentes grupos: El valor Teórico es el primero, el más alto del perfil, sin embargo de acuerdo a los baremos del test tiene un nivel medio; en el segundo grupo están los valores Económico, Estético, Político y Social con puntuaciones similares e intermedias; al final en puntuación y en orden el valor Religioso.

Tabla 4. Prueba de comparación de medias de Tukey.

\begin{tabular}{ccc}
\hline Valores & Media & Grupos \\
\hline Teórico & 51.883 & $\mathrm{~A}$ \\
Económico & 41.428 & $\mathrm{~B}$ \\
Estético & 39.350 & $\mathrm{~B}$ \\
Político & 38.811 & $\mathrm{~B}$ \\
Social & 38.561 & $\mathrm{~B}$ \\
Religioso & 29.967 & $\mathrm{C}$ \\
\hline
\end{tabular}

Valor critico de Rango Estudentizado=4.04.

Diferencia Minima Significativa 2.95 


\subsection{Correlaciones entre los dos perfiles}

Todas las correlaciones de Pearson (Tabla 5) fueron significativas $\left(^{*}\right)$ o altamente significativos (**). Hay una gran relación entre las Dimensiones Organizacionales y los Valores de los Académicos del CP, destacan dos por corresponder a los valores extremos y ser positivas: Estructura - Teórico y Responsabilidad - Religioso, única altamente significativa. Las demás exceptuando Político - Compromiso, son inversas.

Tabla 5. Análisis de correlación de Pearsons (=0.05) entre CO y Valores

\begin{tabular}{llll}
\hline Relaciones entre: & P & Pr>F & Significancia \\
\hline Estructura - Estético & $-32.5 \%$ & 0.032 & $*$ \\
Estructura - Teórico & $28.3 \%$ & 0.057 & $*$ \\
Estructura - Económico & $-34.2 \%$ & 0.030 & $*$ \\
Estándares - Estético & $-38.0 \%$ & 0.023 & $*$ \\
Estándares - Teórico & $-35.6 \%$ & 0.012 & $*$ \\
Estándares - Social & $-29.9 \%$ & 0.051 & $*$ \\
Responsabilidad - Religioso & $\underline{43.6 \%}$ & $\underline{0.002}$ & $* *$ \\
Responsabilidad - Político & $-34.6 \%$ & 0.027 & $*$ \\
Compromiso - Político & $31.6 \%$ & 0.017 & $*$ \\
Reconocimiento - Político & $-33.6 \%$ & 0.035 & $*$ \\
\hline
\end{tabular}

\section{Análisis de resultados}

Aun cuando el Perfil de CO señala tres dimensiones más altas que las otras tres (Tabla 2), ninguna alcanza el límite inferior del nivel más alto (70-100). De acuerdo a los ítems y los criterios de los autores de la escala, el perfil revela preponderancia de actitudes indefinidas o ambiguas, que indican amorfia y división de percepciones en actitudes decisivas para el buen funcionamiento de cualquier organismo social.

El Perfil de Valores de acuerdo a los baremos internacionales de la escala se define como medio-bajo; describe baja intensidad y claridad en los valores de las personas integrantes de la muestra. Estos resultados son semejantes a los obtenidos, por (Mercado, 2004; Pérez-Castro, 2010) - con otros instrumentos - en la academia y docencia. Los autores alertan sobre la precariedad de valores que se difunden en la educación superior por los limitados o ausentes valores en el profesorado, en este artículo nos sumamos a la llamada de atención de estos investigadores.

El valor más alto del perfil (51.88) es el Teórico, describe intereses científicos, coherente con el tipo de institución estudiada. Se trata de intereses abstractos, analíticos y de reflexión, orientaciones científicas de comprensión, más que de aplicación inmediata. El Valor Religioso es el más bajo, funciona como contraparte lógica del valor más alto. De acuerdo a la Teoría de la Personalidad de Allport, el valor más alto define a la persona, en este caso al grupo, los demás son rasgos se consideran complementarios. 


\subsection{Dinámica entre Clima Organizacional y Valores}

Las dimensiones Estructura y Estándares tienen relaciones con cuatro de los seis valores de la escala respectiva, solo con el Valor Teórico existe relación positiva (Tabla 5). La primera dimensión identifica la forma de distribución de la autoridad y jerarquía en cualquier organismo social, así como actitudes y comportamientos que se derivan de ella. La estructura del CP descrita en su organigrama es vertical y se relaciona de manera positiva con los Valores Teóricos, por ello se entiende que la verticalidad de la estructura está apoyada por Valores que responden al "deber ser;" conceptos abstractos de autoridad, orden y disciplina. Las personas del cuerpo académico, cuyo valor principal es el teórico aceptan una estructura jerárquica, que "ponga orden, acepte y respete las aportaciones científicas". Las relaciones inversas de Estructura son con los Valores Estético y Económicos, señala que: A mayores Valores Estéticos y Económicos, más rechazo a la estructura vertical del CP.

La dimensión Estándares se refiere a los niveles de rendimiento que el personal académico y estudiantes tienen que sostener y mejorar, a la normatividad y características requeridas a los nuevos miembros de la comunidad académica. Identifica también sentimientos de orgullo y pertenencia a la institución y satisfacción laboral. Los contenidos de esta dimensión se relacionan en forma inversa con los Valores Estéticos, Teóricos y Sociales, lo que se traduce como a más intensidad de estos valores en los académicos y académicas, menos interés y atención a los índices de productividad y competitividad; posiblemente los perciben como distractores. Del contenido de los ítems se entiende que a mayores Estándares menos orgullo y sentido de pertenencia a la institución, satisfacción laboral y sentido social de su profesión.

Las relaciones de las Dimensiones Responsabilidad, Reconocimiento, Compromiso y Apoyo; y con los Valores del personal académico, plantean contradicciones o incongruencias. Esto se debe: a) El nivel medio del perfil describe comportamientos con preponderancia de actitudes indefinidas, o ambiguas, que indican amorfia y división de percepciones (Litwin y Stringer, 2003); y b) por las puntuaciones tan bajas de los valores con los que se relacionan.

La relación Responsabilidad - Religioso es la más alta y positiva, y Responsabilidad - Político la más baja y negativa (Tabla 5). Así se identifica a dos tipos de personas cuyos valores plantean polos en el conjunto del personal académico. Por la revisión de ítems y comentarios de las personas participantes se entiende que los integrantes del cuerpo académico que tienen altos valores Religiosos no forman grupo ni buscan conseguir beneficios, son respetuosos y confiables en sus compromisos. Los que tienen altos Valores Políticos se interesan en posiciones de representación, son sociables, fácilmente hacen alianzas y tienden a formar grupos. En ambos casos hay tendencia a no percibir la co-responsabilidad de parte de las autoridades en el desarrollo de las actividades académicas; o percibirla de manera ambivalente, lo que se explica por la relación positiva Compromiso - Valores Políticos.

Las dimensiones Compromiso y Reconocimiento también se relacionan con el Valor Político, de manera positiva la primera e inversa con la segunda. La Dimensión Compromiso explora la percepción de la forma en que se relacionan los niveles altos de la estructura con mandos subsiguientes. En el caso del CP no hubo claridad de percepción, por ejemplo algunas personas manifestaron conocer quiénes son los directivos, pero no quien o quiénes toman las 
decisiones, por lo que no identifican que tanta co-responsabilidad pueden esperar de ellos en la realización de sus actividades. La relación de la Dimensión Reconocimiento con el Valor Político reveló, por un lado, las formas de la institución de valorar los logros académicos, por otro lado la necesidad generalizada entre académicos y académicas de ser reconocidos, en especial las personas con valores políticos importantes.

La Dimensión Apoyo no se relaciona con ningún Valor, lo que parece coherente con las percepciones registradas sobre la falta de co-responsabilidad y el reconocimiento hacia su trabajo que sienten los académicos por parte de los directivos. Con relación a sus compañeros comentan: "[...] te tienes que cuidar de todos; [...] si pueden, te taponean el camino"; expresiones que denotan comportamientos de agresión generalizada e imitación de lo que sienten recibir de las autoridades. Esta información se suma a la producida por otros estudios que hablan del nivel bajo o ausencia de valores en profesores de nivel medio y superior (ver inciso de Bases Teóricas, Valores).

\section{Conclusiones}

Con el apoyo de los resultados y una consulta a los documentos básicos de la institución; en este apartado se responde la pregunta de investigación. En primer lugar se puntualiza que las capacidades y limitaciones organizacionales del CP están involucradas con las del personal académico; y que los comportamientos organizacionales del $\mathrm{CP}$ se rigen por los principios, conceptos y definiciones formales u oficiales que lo delinean. Recuperado de http:// www.colpos.mx/web11/index.php/acerca-de/funcionamiento

\section{1 Capacidades y limitaciones organizacionales}

Las dimensiones del Clima Organizacional consideradas en esta investigación, describen comportamientos estructurados, determinados con influencia del valor más alto del perfil respectivo. La tendencia teórica a niveles de abstracción imprime su sello tanto la redacción de sus documentos formales Misión, Visión, Objetivos Estratégicos y Funcionamiento; como en los comportamientos de sus integrantes. Situación explicable por el carácter obligatorio que se establece entre documentos formales y miembros de un organismo. En los documentos fundamentales del CP se encontró un reflejo del perfil de CO que revela poca claridad:

- El CP se presenta en su misión como "[...] institución educativa, en su Visión como "[...] institución pública cuyas actividades sustantivas son educación, investigación y vinculación[...]" (COLPOS, 2013). Sin embargo, en la descripción de su funcionamiento incluye conceptos del orden empresarial que impulsó la administración federal de los años 2000 - 2006: "El Colegio de Postgraduados cuenta con órganos ejecutivos tanto en su corporativo como en sus campus, y con órganos de coordinación al nivel corporativo," (COLPOS, 2013).

- En los objetivos estratégicos del CP se incluyen aspectos para los cuales no cuenta con las estrategias, recursos humanos, ni comprensión de lo que se requiere para 
lograrlos. El su primer objetivo el CP declara dedicarse a "educar y formar personas creativas, innovadoras y con sentido humanista, que atiendan las necesidades agroalimentarias de la sociedad en un contexto de desarrollo sustentable" (COLPOS, 2013). Por haber sido fundado, integrado, atendido y dirigido por profesionales de las ciencias agronómicas, se considera expertos para la segunda expresión del objetivo, no así para la primera. En este objetivo se aprecia también un desajuste entre el self real y el self ideal del CP. Como base empírica para esta aseveración se cuenta con la evidencia de un conflicto prolongado en la institución y el perfil medio - bajo de Valores que obtuvo en esta investigación.

- En su cuarto objetivo, el COLPOS (2013) persigue "mejorar la calidad de vida de la sociedad y retroalimentar las actividades académicas a través de la vinculación;" la redacción revela la limitación de no comprender la función social de la institución y el deseo de resolver un problema nacional muy amplio, así la primera expresión del cuarto objetivo se vuelve un deseo, porque no le compete a una sola entidad de la estructura nacional.

La estructura vertical del CP revela predominio del ejercicio de la autoridad en pocas personas, así como la exclusión de esta actividad a la mayoría del personal académico, aunque las decisiones sean sobre el trabajo que este desarrolla. Tal organización genera disminución de la responsabilidad en las actividades comunes, aumentando las personas que cumplen con lo estrictamente necesario, evitando verse involucrados en alguna comisión o propuestas de tipo grupal.

\subsection{Limitaciones del Cuerpo Académico}

Por ser los perfiles de $\mathrm{CO}$ y Valores correspondientes a personal altamente calificado en la academia, la mayoría con estudios de posgrado en el extranjero, lo que implica un nivel de inteligencia alto, llama la atención la disminución de la capacidad perceptual y la baja intensidad de sus valores. Hechos que hablan de disminución del potencial humano, lo que induce a pensar que el origen, en el grupo estudiado, sean estados depresivos.

En la consulta bibliográfica se encontraron investigaciones de académicos de universidades y posgrado, que reportan el Síndrome de Desgaste Emocional (SDE) o Burn-out académico, producido por exceso de estrés laboral, sobre carga de trabajo y ejecución de roles múltiples y falta de satisfacción laboral. El síndrome también se está reportando en América Latina en diversas universidades (Gil-Monte Peiró, 2000, citados por Caballero, Hederich y Palacio, 2010; Maslach, Schaufeli y Leiter 2001; Guerrero, 2003; Gil-Monte, Nuñez y Selva-Santoyo, 2006; Marrau, 2004; Magaña y Sánchez, 2008) entre otros.

Otra limitante en el cuerpo académico del CP, la constituyen dos comportamientos, por un lado, haber asimilado comportamientos evasivos de responsabilidades, y manejar su agresión de manera difusa, esto sin darse cuenta ni entender como cancelarlos, porque la estructura vertical lo permite, los directivos y los académicos cercanos a ellos han sido ejemplo en estas dos acciones. Se entienden como manifestaciones de frustración, y se 
traduce también, en un obstáculo para el trabajo de los propios compañeros. En conjunto, actitudes y comportamientos, Constituyen un mecanismo complejo de manejo de autoridad, el dañar a otros colegas es poder hacer lo que ha recibido y ejercer poder.

Se puede decir que la tendencia agresiva se manifestó, en este estudio, desde la negativa de algunos miembros del personal académico a responder las escalas para participar en la investigaciòn. El factor común de las negativas se hizo manifiesto, al no ser suficiente la posibilidad de no asistir a la cita, lo hicieron con manifestaciones de rechazo, molestia y enojo, descalificando lo que se hacía. El estudio de (Chang, et. al. 2011) identifica y explora comportamientos semejantes a los antes descritos entre los empleados destinados a la prevención de violencia, concluyendo que la percepción del Clima Organizacional agresivo y la exposición previa a la violencia, aumentan el estrés y disminuye la motivación al trabajo con tendencia a reproducir agresividad.

\section{Sugerencias}

8. 1. Es necesario que los nuevos directivos y cuerpo académico conozcan los resultados de la investigación y se propongan trabajar sobre las siguientes sugerencias:

a. Es necesario que se identifiquen y conozcan las consecuencias de diversas incongruencias entre los documentos oficiales y las capacidades para implementarlos.

b. Conviene que los errores identificados sean eliminados en el diseño de la nueva estructura y se rescate la participación del cuerpo académico en las decisiones y responsabilidades de la institución.

c. Es impotante que se incida en la promoción de procesos de desarrollo de capacidades y atención al personal académico, facilitándoles entrenamiento en comunicación directa, clara y efectiva orientados progresivamente a la integración de equipos de trabajo y la aceptaciòn de responsabilidades acadèmicas.

d. Conviene impulsar los medios apropiados para que se produzcan cambios de comportamiento trabajando en forma paralela el cambio de estructura y reglamentación, y de actitudes hacia el trabajo; en especial cooperación y ejercicio de la autoridad, como responsabilidad para el desarrollo institucional.

e. Es importante tambièn atender por medio de procesos grupales cualquier requerimiento de información y cambio de actitudes.

8.2 Conviene confirmar el problema detectado de Síndrome de Depresión Emocional, empleando instrumentos específicos, para para, con base a los resultados y las reacciones en los procesos de comunicación, diseñar grupos de análisis y comprensión del problema, así como difusión de medidas de atención de sañid física y psicológicae incluir en estos procesos a profesionales de la salud mental con el objetivo de facilitar consultas individuales, que serán voluntarias y fuera de los campus del CP. 


\section{Referencias bibliográficas}

Acosta, A. (2005). Departamentalización y Contexto Organizacional: la experiencia de la Universidad de Guadalajara. Revista Electrónica de Investigación Educativa, 7(1), 1-18.

Allport, G. W., Vernon, P. E. \& Lindzey, G. (1970). Study of values. (Revised third ed.). Chicago: The Riverside Publishing Company.

Arciniega, L. \& González, L. (2000). Desarrollo y validación de la escala de valores hacia el trabajo EVAT 30. Revista de Psicología Social, 15(3), 281-296.

Barba, B. (2005). Educación y valores. Una búsqueda para reconstruir la convivencia Revista Mexicana de Investigación Educativa, 10(24), 9-14.

Bárcenas, R. (2009). Pertinencia: una dimensión de la calidad de la enseñanza. Valor intrínseco en las relaciones encaminadas al consenso de las normas y los contenidos curriculares. Tiempo de Educar, 10(20), 349-378.

Brewczynski, J. \& MacDonald, D. (2006). Confirmatory Factor Analysis of the Allport and Ross Religious Orientation Scale with a Polish Sample. International Journal for the Psychology of Religion, 16(1), 63-76.

Burton, R. \& Obel, B. (2004). Strategic organizational diagnosis and design. The dynamics of fit. [Estrategia de diagnóstico y diseño organizacional. Las dinámicas del ajuste]ף. Cambridge: Cambridge University Press.

Caballero, C. Hederich, C. \& Palacio, J. (2010). El burnout académico: delimitación del síndrome y factores asociados con su aparición. Revista Latinoamericana de Psicología, 42(1), 131-146.

CACM. Comité Académico del Campus Montecillo, CP. (2005). Causas del retraso en el periodo de graduación de estudiantes del Colegio de Posgraduados. Encuesta a ex alumnos y alumnos. Colegio de Posgraduados.

Chang, C., Eatough, E., Spector, P. \& Kessler, S. (2011). Violence-prevention climate, exposure to violence and aggression, and prevention behavior: A mediation model. [Clima de prevención de la violencia, la exposición a la violencia y la agresión, comportamiento de prevención: Un modelo de mediación]. Journal of Organizational Behavior, 33(5), 657677.

COLPOS. Colegio de Posgraduados (2013). Misión, Visión y Objetivos. México: Colegio de Postgraduados. Recuperado de http://www.colpos.mx/web11/index.php/acerca-de/ mision-vision-y-objetivos.

COLPOS. Colegio de Posgraduados (2013). Funcionamiento. México: Colegio de Postgraduados. Recuperado de http://www.colpos.mx/web11/index.php/acerca-de/funcionamiento

Comisión Mixta Wageningen - México. (2004). Evaluación del Colegio de Postgraduados. Comisión Mixta Colegio de Postgraduados (México) - Universidad de Wageningen (Holanda). INAP, José Reyes Sánchez (Consultor). 
De Miguel, M. (2006). Cambio de Paradigma Metodológico en la Educación superior: exigencias que conlleva la Universidad de Oviedo. Cuadernos de Integración Europea No. 2.

Epitropaki, O. (2012). A multi-level investigation of psychological contract breach and organizational identification through the lens of perceived organizational membership: Testing a moderated-mediated model. [Una investigación de niveles múltiples de incumplimiento del contrato psicológico e identificación organizacional a través de la lente de la membresía organizacional percibida: Prueba de un modelo de moderación mediada] Journal of Organizational Behavior. 34(1), 65 - 86.

Esquivel, F. (2008). Acercamiento a la valoración de la formación académica de la Universidad de Costa Rica y su vínculo con la realidad nacional. Revista Educación 32(2), 13-28.

García, J. \& Pérez, E. (2005). Razonamiento Moral y valores: estudio de sus relaciones en un grupo de universitarios españoles. Revista Latinoamericana de Psicología, 37(1), 131-148.

Gil-Monte, P. \& Peiró, J. (2000). Síndrome de quemarse por el trabajo (síndrome de Burnout), aproximaciones teóricas para su explicación y recomendaciones para la intervención. Recuperado de: http://www.psicologiacientifica.com.

Gil-Monte, P., Núñez, E. \& Selva-Santoyo, Y. (2006). Relación entre el Síndrome de Quemarse por el Trabajo (Burnout) y Síntomas Cardiovasculares: Un Estudio en Técnicos de Prevención de Riesgos Laborales. Interamerican Journal of Psychology, 40(2), 227-232.

González, V. \& Rendón, G. (1986). Análisis de las actividades profesionales realizadas por los egresados de Colegio de Posgraduados (1959-1983). Ed. Colegio de Posgraduados.

González, V. \& Rendón, G. (1999). Estudio sobre mercado laboral de los egresados y ajuste curricular de los programas académicos del Colegio de Posgraduados, mediante una encuesta por muestreo. Colegio de Posgraduados.

Guerrero, E. (2003). Análisis pormenorizado de los grados de burnout y técnicas de afrontamiento del estrés docente en profesorado universitario. Anales de Psicología, 19(1), 145-158.

Gupta, A. (2008). Organizational Climate Study, Organizational Traineeship. [Estudio de Clima Organizacional, Prácticas Organizacionales]. Segmento, PRM 28055, Institute of Rural Management, Anand.

Hirsch, A. (2008) Formación en ética profesional y los profesores de posgrado de la Universidad Nacional Autónoma de México. Revista Electrónica de Investigación Educativa [Número Especial]. Recuperado de: http://redie.uabc.mx/NumEsp2/contenido-hirsch3.html

Izarra, D. \& Escobar, F. (2007). Pertinencia de la investigación en los estudios de postgrado de la UPEL-IMPM. Investigación y Postgrado, 22(2), 165-186.

Jones, J. M., Chair, S. D., Cochran, M. F., Gaertner, S., Mendoza-Denton, R., Shih, M. \& Derald-Wing, S. (2012). APA Presidential. Task Force on Reducing and Preventing 
Discrimination Against and Enhancing Benefits of Inclusion of People Whose Social Identities Are Marginalized in U.S. Society. [Grupo de Trabajo sobre la reducción y la prevención de la discriminación y la mejora de los beneficios de la inclusión social de personas cuyas identidades se marginan de la sociedad EUA] Final Report. American Psychological Association.

Koene, B., Vogelaar, A.. \& Soeters, J. (2002). Leadership effects on organizational climate and financial performance: Local leadership effect in chain organizations. [Los efectos del liderazgo sobre el clima organizacional y el desempeño financiero: Efecto de liderazgo local en las organizaciones de la cadena]. The Leadership Quarterly, 13(3), 193-215.

Lemmergaad, J. (2003). Tolerance for Ambiguity. The intersection Between Ethical Climate. Psychological Climate, and Ethnic Diversity. [Tolerancia a la ambigüedad. La intersección entre el clima ético. Clima psicológico, y la diversidad étnica]. Ph. D. dissertation. University y of Southern Denmark.

Litwin, G. \& Stringer, R. (2003). Motivation and organizational climate. [Motivación y clima organizacional]. U.S.A.: Harvard University Press.

Lopera, C. (2004). Antinomias, dilemas y falsas premisas que condicionan la gestión universitaria. Revista Mexicana de Investigación Educativa, 9(22), 617-635.

López, A., Villa, M. \& Badillo, E. (1983). Análisis económico del Plan Zacapoaxtla; Programa de Investigación CE-CEICADAR, escrito No. 3. Colegio de Postgraduaos.

Magaña, D. E. \& Sánchez, P. A. (2008). Síndrome de Desgaste Emocional en Investigadores Mexicanos. Interamerican Journal of Psychology, 42 (2), 353-362.

Mano, R. \& Yiannis, G. (2006). Workplace romances in cold and hot organizational climates: The experience of Israel and Taiwan. [Romances en el lugar de trabajo en climas organizacionales fríos y calientes: La experiencia de Israel y Taiwan]. Human Relations, 59 (1), 7-35.

Marrau, C. (2004). El síndrome de Burnout y sus posibles consecuencias en el trabajador docente. Fundamentos en humanidades, 5 (2), 53-68.

Maslach, C., Schaufeli, W. B. \& Leiter, M. P. (2001). Job Burnout. [Agotamiento por el trabajo]. Annu. Rev. Psychol, 52, 397-422.

Mercado, E. (2004). Los valores y la docencia de los maestros puestos en la escena. Tiempo de Educar, 5(10), 135-158.

Mollis, M. (2002). Un breve diagnóstico de las universidades Argentinas: identidades alteradas. ¿Instituciones del saber o supermercados de conocimientos? CLACSO, Argentina. Recuperado de: http://bibliotecavirtual.clacso.org.ar/ar/libros/mollis/mollis.pdf

Muñoz, J. M., Ríos, M. P. \& Abalde, E. (2002). Evaluación docente vs evaluación de la calidad. Revista Electrónica de Investigación y Evaluación Educativa, 8 (2), 103-134.

Padilla, L., Jiménez, L. \& Ramírez, M. (2008). La satisfacción con el trabajo académico: Motivaciones y condiciones del entorno institucional que la afectan. El caso de una 
universidad pública estatal. Revista Mexicana de Investigación Educativa, 13 (38), 843865.

Pedró, F. (2006). Un diagnóstico de la situación del profesorado en España desde una perspectiva comparativa. Revista de Educación, 340, 243-264.

Pérez-Castro, J. (2010). Elementos para la reflexión en torno a la ética profesional de los estudiantes de posgrado. Revista Electrónica de Investigación Educativa, 12 [Número Especial]. Recuperado de: http://redie.uabc.mx/NumEsp2/contenido-perezcastro.html

Pierce, J. D., Cohen, A. B., Chambers, J. A. \& Meade R. M. (2007). Gender differences in death anxiety and religious orientation among US high school and college students. [Diferencias de género en ansiedad ante la muerte y la orientación religiosa, en estudiantes de una escuela secundaria y universitarios en EUA]. Mental Health, Religion \& Culture., 10 (2)143-150.

Polanco, A. (1992). Análisis para el Desarrollo Institucional del Colegio de Posgraduados en Ciencias Agrícolas. Informe Técnico de publicación restringida. Colegio de Postgraduados. México.

Porter L. (2004). La planeación de la autoridad, la planeación de la libertad: inconsistencias e incompatibilidades del programa integral de fortalecimiento institucional. Revista Mexicana de Investigación Educativa, 9(22), 585-615.

Pümpin, C. \& García, S. (1993). Estrategia Empresarial. Como implementar la estrategia en la empresa. Madrid, España: Ed. Díaz de Santos, S.A.

Quinn, R. E. \& Rohrbaugh, J. (1983). A spatial model of effectiveness criteria: Towards a competing values approach to organizational analysis. [Un modelo espacial de criterios de eficacia: Hacia un enfoque de valores por competencia para el análisis organizacional]. Management Science, 29, 363-377.

Quintero, J. E., Corrales, V., Martínez, R. \& Aréchiga, G. (2010). El cambio conducido en la universidad: la percepción de los académicos. Revista de la Educación Superior, 39(155), 27-42.

Rodríguez, J. R., Urquidi, L. E. \& Mendoza, G. (2009). Edad, producción académica y Jubilación en la Universidad de Sonora Una primera exploración. Revista Mexicana de Investigación Educativa, 14 (41), 593-617.

Salas, F. (2007). Educación e Investigación y Desarrollo en América Latina: Los últimos treinta años. Educación, 31(2), 29-43.

Sánchez, Q. C. (1984). Las estructuras institucionales que promueven el desarrollo rural y las personas que las ejecutan. Revista Agrociencia. (69) 35 -44

Sánchez, Q. C., Vázquez, D. \& Aguilar, S. (1994). Análisis Organizacional en el Sector Rural. Centro de Estudios del Desarrollo Rural. México: Colegio de Postgraduados.

Santos, J., Muñoz, A., Juez, P., \& Cortiñas, P. (2003). Diseño de encuestas para estudios de mercado. Técnicas de Muestreo y Análisis Multivariante. Madrid: Editorial Centro de Estudios Ramón Areces, S.A. 
Schein, E. H. (1988). La Cultura Empresarial y Liderazgo. Barcelona: Editorial Plaza \& Janes,

Schein, E. H. (2010). Culture and Leadership. [Cultura y Liderazgo] $4^{\mathrm{a}}$ Edition, Published by Wiley \& Sons.

Schulte, M., Ostroff, C. \& Kinicki, A. (2010). Organizational climate systems and psychological climate perceptions: A cross-level study of climate-satisfaction relationships. [Sistemas de clima organizacional y la percepción del clima psicológico: Un estudio transversal de las relaciones del clima satisfactorio]. Journal of Occupational and Organizational Psychology, 79 (4), 645-671.

Starks, A. (2005). Diagnóstico del proceso de cambio y del clima organizacional en el Colegio de Posgraduados. Informe final con recomendaciones de acciones de mejora. México: Valora Consulting.

Stringer, R. (2002). Leadership and Organizational Climate. "Liderazgo y Clima Organizacional" Janes Barcelona: Prentice Hall Series in Organization Develpment. 